\title{
NEW APPROACH TO COMPUTE ACCURATELY THE ENTROPY GENERATION DUE TO NATURAL CONVECTION IN A SQUARE CAVITY
}

\author{
Saadoun Boudebous ${ }^{*}$, Nawal Ferroudj ${ }^{2}$ \\ ${ }^{1}$ Faculty of Sciences and Applied Sciences, University of Larbi Ben M'hidi, Oum el Bouaghi, Algeria \\ ${ }^{2}$ Laboratory of Biotechnology, National Higher School of Biotechnology (ENSB), Constantine 3, Algeria
}

\begin{abstract}
The idea to carry out an exercise to compare the calculation of entropy generation for unsteady natural convection in a square cavity with vertical sides that are maintained at different temperatures was motived by the observation, in the literature, of inaccurate or often erroneous results concerning the values of this significant physical entity. It then appeared necessary to reconsider this problem in order to ensure its consistent assessment. The new approach that we propose allows a direct access to the value of the entropy generation by considering the exact values of the thermophysical properties of the working fluid, which depends on the Prandtl and the Rayleigh numbers.
\end{abstract}

\footnotetext{
* Corresponding author: s.boudebous@gmail.com
} 


\section{Introduction}

It is well established that convective heat transfer processes are always accompanied by a loss of energy commonly referred to as "Entropy Generation" in the scientific literature [1]. Accurately determining the entropy generation in applied thermal engineering is the indispensable prerequisite for the design of different types of thermal exchanging devices [2]. Entropy generation has been and continues to be the subject of many research activities over the past decades, as shown by the significant review articles by Oztop and Al-Salem [3], Sciacovelli et al. [4], and more recently by Kumar et al. [5], and Cai et al. [6]. Solving the entropy generation equation during fluid flows in a natural convection regime, which will be developed later, requires the calculation of a coefficient called "the irreversibility distribution ratio", oftentimes symbolized by the Greek letter $\varphi$.

One real problem lies in an accurate determination of the irreversibility distribution ratio value. Until now, the computing of the relevant value of this ratio has not yet been well established and the gap between the values proposed by different authors can be quite significant. Hussein et al. [7], and Oztop et al. [8], present and discuss the results for entropy generation without specifying any value of the irreversibility ratio. Shavik et al. [9], Yejjer et al. [10], Jassim et al. [11] and Seyyedi et al. [12], impose values of the same ratio ranging from $10^{-3}$ to $10^{-5}$ with no explanation. In the same way, Erbay et al. [13, 14], specify values which increase linearly with the Rayleigh number $(\mathrm{Ra})$, starting at $10^{-13}$ for $\mathrm{Ra}=10^{2}$ to reach $10^{-9}$ for $\mathrm{Ra}=10^{6}$, with constant steps of 10 . Rathnam et al. [15], use an order of magnitude analysis of parameters to evaluate the same ratio at $10^{-3}$. Magherbi et al. [16], Ilis et al. [17], De C. Oliveski et al. [18], and Bouabid et al. [19], consider this coefficient as an investigative parameter in the same way as the Rayleigh, or Prandtl numbers for example in [16], $\varphi$ varies from $10^{-4}$ to $10^{-1}$ and in [17-18-19], $\varphi$ varies from $10^{-4}$ to $10^{-2}$. However, we would also point out that, until very recently (05 February 2021) Karki et al. [20] investigates the exergy analysis of Rayleigh-Benard natural convection by varying the irreversibility ratio $\varphi$ between $10^{-2}$ and $10^{-5}$. Finally, it must be stressed that we identified a numerical study of natural convection and entropy generation reported by Khorasanizadeh and Nikfar [21], in which they have doubts about the fact that the irreversibility distribution ratio $\varphi$ remains constant while at the same time Rayleigh number varies considerably. The values suggested by these authors vary according to the Rayleigh number and are certainly more important than such as the ones already proposed but they are still far from reality, and above all, there is no propose that set forth how these values were concretely obtained.

Based on the aforementioned brief review of the literature, which revealed many shortcomings in the determination of the exact value of the irreversibility distribution ratio $\varphi$, that we propose a new pragmatic approach in order to estimate the fair value of the entropy generation in confined spaces.

\section{Problem description}

The benchmark problem considered here is the differentially heated square cavity problem depicted in Figure 1. It is similar to those referenced by Magherbi et al. [16], Ilis et al. [17], De C. Oliveski et al. [18], and Bouabid et al. [19]

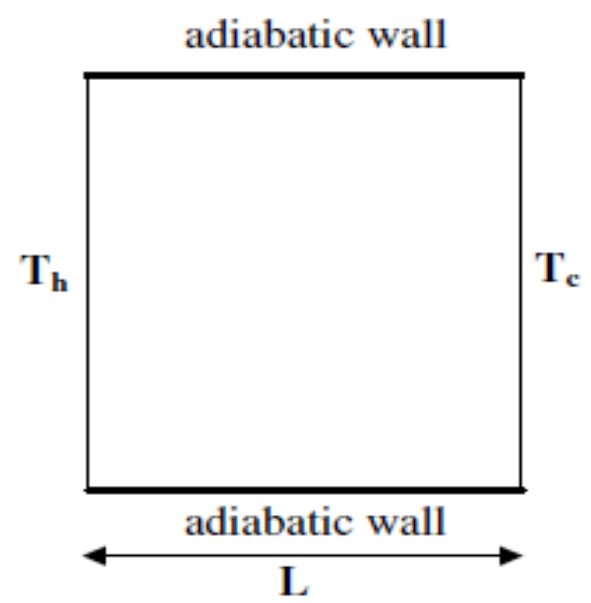

Fig. 1. A schematic view of the benchmark problem [16].

\section{Mathematical formulation}

The stream function-vorticity $(\psi, \omega)$ formulation is used to express the dimensional governing equations for the laminar and unsteady state natural convection in Cartesian coordinates $\mathrm{x}$ and $\mathrm{y}$.

$$
\left.\begin{array}{l}
\frac{\partial^{2} \psi}{\partial x^{2}}+\frac{\partial^{2} \psi}{\partial y^{2}}=-\omega \\
\frac{\partial \omega}{\partial t}+u \frac{\partial \omega}{\partial x}+v \frac{\partial \omega}{\partial y}=v\left(\frac{\partial^{2} \omega}{\partial x^{2}}+\frac{\partial^{2} \omega}{\partial y^{2}}\right)+g \beta \frac{\partial T}{\partial x} \\
\frac{\partial T}{\partial t}+u \frac{\partial T}{\partial x}+v \frac{\partial T}{\partial y}=\alpha\left(\frac{\partial^{2} T}{\partial x^{2}}+\frac{\partial^{2} T}{\partial y^{2}}\right) \\
\text { here } u=\frac{\partial \psi}{\partial y}, v=-\frac{\partial \psi}{\partial x}, \omega=\frac{\partial v}{\partial x}-\frac{\partial u}{\partial y}
\end{array}\right\}
$$

The numerical resolution of the previous system of equations requires the following initial and boundary conditions:

At $\tau=0$ and for whole space.

$\psi=\omega=0, T=T_{0}+\Delta T\left(0.5-\frac{x}{L}\right) \quad 0 \leq x \leq L$

At $\tau>0$

$\psi=0, \omega=-\frac{\partial^{2} \psi}{\partial x^{2}}$ and $\mathrm{T}=\mathrm{Th}$ at left wall

$\psi=0, \omega=-\frac{\partial^{2} \psi}{\partial x^{2}}$ and $\mathrm{T}=\mathrm{Tc}$ at right wall

$\psi=0, \omega=-\frac{\partial^{2} \psi}{\partial y^{2}}$ and $\frac{\partial T}{\partial y}=0$ at bottom and top walls. 
The average Nusselt number $\overline{n u}$ can be expressed, on the basis of the dimensional variables, as follows:

$\overline{n u}=-\left.\frac{1}{\Delta T} \int_{0}^{L} \frac{\partial T}{\partial x}\right|_{x=0} d y$

The local entropy generation is given by [21]:

$$
\begin{aligned}
& s_{\text {gen }}=\underbrace{\frac{k}{T_{0}^{2}}\left\{\left(\frac{\partial T}{\partial x}\right)^{2}+\left(\frac{\partial T}{\partial y}\right)^{2}\right\}}_{s_{t h}} \\
& +\underbrace{\frac{\mu}{T_{0}}\left\{2\left(\frac{\partial u}{\partial x}\right)^{2}+2\left(\frac{\partial v}{\partial y}\right)^{2}+\left(\frac{\partial u}{\partial y}+\frac{\partial v}{\partial x}\right)^{2}\right\}}_{s_{f f}}
\end{aligned}
$$

The total entropy generation is the integral over the system volume of the local entropy generation:

$$
\overline{s_{\text {gen }}}=\frac{1}{\vartheta} \int_{\vartheta} s_{\text {gen }} d \vartheta
$$

Another parameters that characterizes the irreversibilities distribution are the local and the average Bejan number defined respectively as [16]

$$
b e=\frac{s_{t h}}{s_{g e n}} \quad \overline{b e}=\frac{1}{\vartheta} \int_{\vartheta} b e d \vartheta
$$

The dimensionless form of the governing equations may be written with following dimensionless variables:

$$
\left.\begin{array}{l}
X, Y=\frac{1}{L}(x, y), \quad \Theta=\frac{T-T_{0}}{T_{h}-T_{c}}, \quad U, V=\frac{L}{\alpha}(u, v), \quad \tau=\frac{\alpha}{L^{2}} t \\
\frac{\partial^{2} \Psi}{\partial X^{2}}+\frac{\partial^{2} \Psi}{\partial Y^{2}}=-\Omega \\
\frac{\partial \Omega}{\partial \tau}+U \frac{\partial \Omega}{\partial X}+U \frac{\partial \Omega}{\partial Y}=\operatorname{Pr}\left(\frac{\partial^{2} \Omega}{\partial X^{2}}+\frac{\partial^{2} \Omega}{\partial Y^{2}}\right)+\operatorname{RaPr} \frac{\partial \Theta}{\partial X} \\
\frac{\partial \Theta}{\partial \tau}+U \frac{\partial \Theta}{\partial X}+U \frac{\partial \Theta}{\partial Y}=\frac{\partial^{2} \Theta}{\partial X^{2}}+\frac{\partial^{2} \Theta}{\partial Y^{2}} \\
\text { Where } U=\frac{\partial \Psi}{\partial Y}, V=-\frac{\partial \Psi}{\partial X}, \Omega=\frac{\partial V}{\partial X}-\frac{\partial U}{\partial Y} \\
\operatorname{Pr}=\frac{v}{\alpha}, \quad \operatorname{Ra}=\frac{g \beta \Delta T P r}{v^{2}} L^{3}
\end{array}\right\}
$$

The average Nusselt number $\overline{N u}$ can be expressed, on the basis of the dimensionless variables, as follows:

$\overline{N u}=-\left.\int_{0}^{1} \frac{\partial \Theta}{\partial X}\right|_{X=0} d Y$

And the local entropy generation, with the same dimensionless variables is given by:

$$
\begin{aligned}
& S_{\text {gen }}=\underbrace{c_{1}\left(\frac{\partial \Theta}{\partial X}\right)^{2}+\left(\frac{\partial \Theta}{\partial Y}\right)^{2}}_{\text {Sth }} \\
& +\underbrace{c_{2}\left\{2\left(\frac{\partial U}{\partial X}\right)^{2}+2\left(\frac{\partial V}{\partial Y}\right)^{2}+\left(\frac{\partial U}{\partial Y}+\frac{\partial V}{\partial X}\right)^{2}\right\}}_{S_{f f}}
\end{aligned}
$$

The total entropy generation is given by:

$$
\overline{S_{g e n}}=\int_{0}^{1} \int_{0}^{1} S_{g e n} d X d Y
$$

The two irreversibilities coefficient are:

$$
c_{1}=k\left(\frac{\Delta T}{T_{0} L}\right)^{2} \quad c_{2}=\frac{\mu}{T_{0}}\left(\frac{\alpha}{L^{2}}\right)^{2}
$$

The first term in equations (2) and (6) is the local entropy generation due to heat transfer and the second term is the local entropy generation due to fluid friction.

The dimensionless form of Eq. (4) takes the form

$$
B e=\frac{S_{t h}}{S_{g e n}} \quad \overline{B e}=\int_{0}^{1} \int_{0}^{1} B e d X d Y
$$

At this point it must be underlined that the lowercase letters correspond to the dimensional variables and the uppercase letters correspond to the dimensionless variables.

It should be noted that to appraise the total volumetric entropy generation all the authors previously cited use the local entropy generation number defined by:

$$
\begin{aligned}
& N_{S}=\frac{S_{\text {gen }}}{c_{1}}=\left(\frac{\partial \theta}{\partial X}\right)^{2}+\left(\frac{\partial \theta}{\partial Y}\right)^{2} \\
& +\varphi\left[2\left(\frac{\partial U}{\partial X}\right)^{2}+2\left(\frac{\partial V}{\partial Y}\right)^{2}+\left(\frac{\partial U}{\partial Y}+\frac{\partial V}{\partial X}\right)^{2}\right]
\end{aligned}
$$

Where $\varphi$ is the irreversibility distribution ratio defined

as: $\varphi=\frac{c_{2}}{c_{1}}=\frac{\mu T_{0}}{k}\left(\frac{\alpha}{\Delta T}\right)^{2} \frac{1}{L^{2}}$

The total dimensionless entropy generation number is written:

$$
\overline{N s}=\int_{0}^{1} \int_{0}^{1} N s d X d Y
$$

The primary objective of this work is to suggest a method that is mathematically and physically correct for the determination of the irreversibility distribution ratios thus providing the true value of the entropy generation.

\section{Numerical procedure}

\subsection{Discretisation method}

The above-mentioned systems of equations (I) and (II) subject to their respective initial and boundary conditions have been discretized by the finite difference method. Temporal discretization has been achieved using the Runge-Kutta fourth-order method (R.K.4). The convective terms have been discretized with a third-order upwind scheme as proposed by Kawamura et al. [23]. The diffusive terms, as well as the terms including the first derivatives, have been discretized by a fourth-order accurate scheme. An iterative procedure based on the successive Non Linear Over Relaxation method (NLOR) was used to solve the discredited stream function equation. Once the velocities and temperatures have been determined, the average Nusselt number (Eqs.1,5) and the characteristics of the total entropy generation in dimensional and dimensionless variables are computed 
using equations (3), (4), (7), (9), and (12). An in-house FORTRAN code, with a double precision accuracy, has been developed for solving the systems of the discretized equations.

\subsection{Grid independency test}

Numerical tests have been made to ensure the accuracy of results for the grid used in this study. Five grid sizes $(41 \times 41 ; 81 \times 81 ; 101 \times 101 ; 161 \times 161$ and $201 \times 201)$ have been considered. Table 1 shows the convergence of the average Nusselt number and the total entropy generation for $\mathrm{Pr}=0.7$ and $\mathrm{Ra}=103$. It should be noted that the maximum relative error does not exceed $2 \%$ between the grid sizes of $81 \times 81$ and $101 \times 101$ compared to the grid size of $201 \times 201$. Therefore, it was decided to use a non-uniform grid with $101 \times 101$ grid points for all calculations allowing a balance between accuracy and CPU time.

Table 1. Grid independence test: average Nusselt number and total entropy generation for $R a=10^{3}, \operatorname{Pr}=0$.

\begin{tabular}{ccc}
\hline Grid & $\overline{N u}$ & $\overline{S_{\text {gen }}}$ \\
\hline $41 \times 41$ & 1.1196 & 0.16057 \\
$81 \times 81$ & 1.1182 & 0.16034 \\
$101 \times 101$ & 1.1181 & 0.16034 \\
$161 \times 161$ & 1.1179 & 0.16031 \\
$201 \times 201$ & 1.1178 & 0.16030 \\
\hline
\end{tabular}

\subsection{Code Validation}

In order to ensure the effectiveness of the developed code, validation was carried out to compare the values of the average Nusselt number obtained in the classic case of natural convection occurring in a square cavity with differentially heated vertical sides and insulated horizontal walls. Table 2 shows this comparison for different Rayleigh numbers when the Prandtl number is set at 0.7 .

Table 2. Comparison of the present average Nusselt number with available literature

\begin{tabular}{ccccc}
\hline Ra & Ref.[24] & Ref.[25] & Ref.[26] & Present \\
\hline $10^{3}$ & 1.116 & 1.127 & 1.117 & 1.118 \\
$10^{4}$ & 2.238 & 2.245 & 2.246 & 2.249 \\
$10^{5}$ & 4.509 & 4.521 & 4.530 & 4.537 \\
$10^{6}$ & 8.817 & 8.800 & 8.822 & 8.825 \\
\hline
\end{tabular}

In view of this validation, it can be concluded that the computation code developed for this study gives results in accordance with those cited in the literature.

\section{Results and discussion}

\subsection{Details data associated with the benchmark}

Numerical simulations have been performed for different Rayleigh numbers ( $\mathrm{Ra}$ ). The thermo-physical properties of the working fluid, for Prandtl number equal to 0.7 , have been taken from the book by the authors Incropera et al.[27] at the bulk temperature T0 $=350 \mathrm{~K}$ and are listed in Table 3 . Furthermore, the temperature difference $\Delta \mathrm{T}$ are kept constant at $10 \mathrm{~K}$.
Table 3. Thermo-physical properties of air $(\mathrm{Pr}=0.7)$ at $\mathrm{T}_{0}=350 \mathrm{~K}$ expressed in the International System (SI) of units.

\begin{tabular}{|c|c|c|c|c|}
\hline$\rho$ & $\mathrm{k}$ & $\mu$ & $\alpha$ & $\beta$ \\
\hline 0.995 & $3.10-2$ & $2.08210-5$ & $2.9910-5$ & $2.85710-5$ \\
\hline
\end{tabular}

\subsection{The irreversibility ratios calculation method}

It can be seen that the irreversibility ratios $\mathrm{c} 1$ and $\mathrm{c} 2$ in equation (8) are directly proportional to the thermophysical properties of the fluid, to the temperature difference $\Delta \mathrm{T}$, and (perhaps more importantly) to the cavity length L. All these parameters with the exception of the cavity length $\mathrm{L}$ are known for each Prandtl number. This effective length could be evaluated numerically from the Rayleigh number as follow:

$R a=\frac{g \beta \Delta T P r}{v^{2}} L^{3} \Rightarrow L=\sqrt[3]{C^{n t} R a}$

Where $C^{n t}=\frac{v^{2}}{g \beta \Delta T P r}$ is a constant, and $v=\frac{\mu}{\rho}$ is the kinematic viscosity.

This new approach does take into account all these parameters and thus provides a better reflection of the real value of the irreversibility ratios (which are given in table 4), thus providing the true value of the entropy generation.

Table 4. Real values of the irreversibility ratios for different Rayleigh numbers $(\mathrm{Ra})$ and $\mathrm{Pr}=0.7$

\begin{tabular}{cccc}
$\mathrm{Ra}$ & $\mathrm{c}_{1}$ & $\mathrm{c}_{2}$ & $\varphi=\mathrm{c}_{2} / \mathrm{c}_{1}$ \\
\hline $10^{3}$ & $1.434010^{-1}$ & $1.823510^{-9}$ & $1.271610^{-8}$ \\
$10^{4}$ & $3.089610^{-2}$ & $8.464010^{-11}$ & $2.739610^{-9}$ \\
$10^{5}$ & $6.656310^{-3}$ & $3.928710^{-12}$ & $5.902210^{-10}$ \\
$10^{6}$ & $1.434010^{-3}$ & $1.823510^{-13}$ & $1.271610^{-10}$ \\
\hline
\end{tabular}

\subsection{Comparison with Benchmark results}

\subsubsection{Method validation}

The validity of the proposed method has been verified by solving the systems of equations (I) and (II) expressed with the dimensional, and the dimensionless variables respectively.

The comparisons of average Nusselt number, total entropy generation, total entropy generation due to fluid friction and average Bejan number for a Rayleigh number equal to 104 are shown in table 5. As seen, the obtained results are quite similar, because we have taken into account the thermo-physical properties of the fluid (see Table 3 ) and the cavity length L calculated from equation (13).

Table 5. Different parameters obtained from the equations solved with the dimensional and dimensionless variables $\left(\mathrm{Ra}=10^{4}\right)$ 


\begin{tabular}{ll|ll}
\hline \multicolumn{2}{c|}{ Dimensional variables } & \multicolumn{2}{|c}{ Dimensionless variables } \\
\hline$\overline{n u}$ & 2.249050 & $\overline{N u}$ & 2.249070 \\
$\overline{S_{\text {sen }}}$ & $6.94941910^{-2}$ & $\overline{S_{\text {sen }}}$ & $6.94948210-2$ \\
$\overline{S_{f f}}$ & $8.81988210^{-7}$ & $\overline{S_{f f}}$ & $8.82009510-7$ \\
$\overline{b e}$ & 0.999987308 & $\overline{B e}$ & 0.999987308 \\
\hline
\end{tabular}

\subsubsection{Total entropy generation calculation}

The total entropy generation equations (6) and (7) subject to the exact values of the two irreversibilities coefficient $\mathrm{c} 1$ and $\mathrm{c} 2$ given by equation (8) have been numerically solved for different Rayleigh numbers. The obtained results, given in figure 2 , provide a direct measurement of the total entropy generation in the physical domain expressed in $\mathrm{W} / \mathrm{m} 3 \mathrm{~K}$. It should be noted that they are most helpful to the engineers and designers in order to design and optimize different types of equipment involving heat transfer in fluid flows.

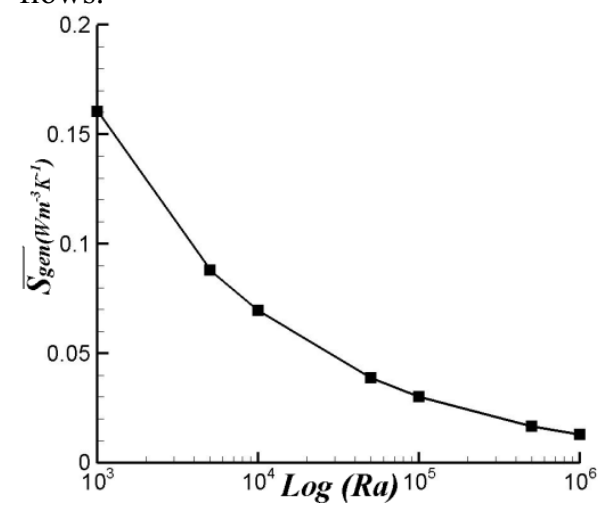

Fig. 2. Total entropy generation $\overline{S_{\text {gen }}}\left(W m^{-3} K^{-1}\right)$ versus the Rayleigh numbers (Ra).

\subsubsection{Total dimensionless entropy generation number}

Total dimensionless entropy generation number versus Rayleigh numbers is shown graphically in Figures 3 and 4. In figure 3 the total dimensionless entropy generation number is computed with an the irreversibility distribution ratio $\varphi$, by taking account of the thermo-physical properties of the fluid (see Table 3 ) and the cavity length $L$ calculated from equation (13), while, in figure 4 the same parameter is computed with an the irreversibility distribution ratio $\varphi$, which is set arbitrarily at $10^{-4}$, according to the approach adopted by Magherbi et al. [16], Ilis et al. [17], De C. Oliveski et al. [18], and Bouabid et al. [19].

We clearly observe some of the numerical differences in solutions between the two approaches for the total dimensionless entropy generation number $(\overline{N s})$. In figure $3 \overline{N s}$ varies on a scale of 1 to 9 with a slightly increasing slope, while in figure $4 \overline{N s}$ varies from 1 to
400. This variation is moderately progressive for $\mathrm{Ra}$ less than $10^{5}$ and becomes exponential afterwards.

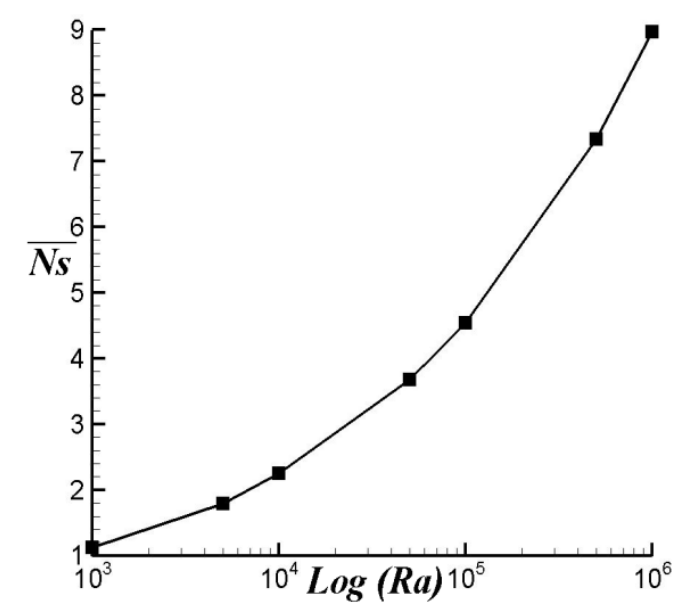

Fig. 3. Total dimensionless entropy generation number versus the Rayleigh numbers (Ra) according to our approach.

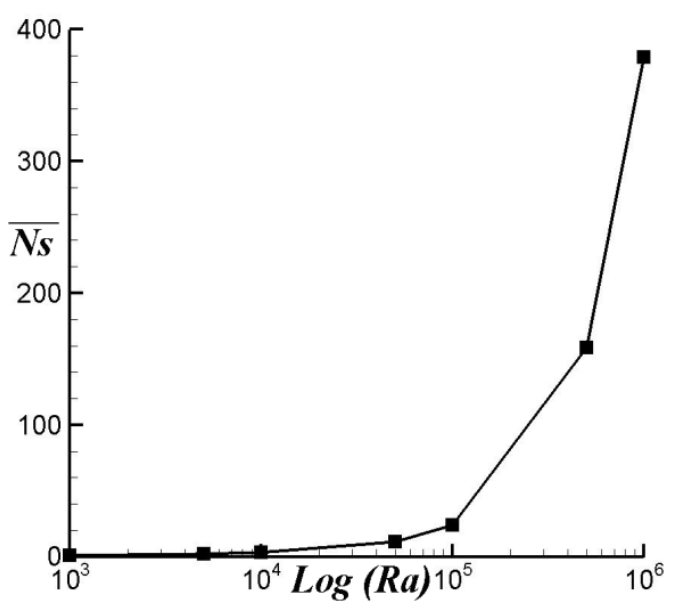

Fig. 4. Total dimensionless entropy generation number versus the Rayleigh numbers (Ra) according to the approach referenced in [16-19].

\subsubsection{Formatting the title}

The evolution of the average Bejan number is shown in Figure 5 and, 6 respectively, for different Rayleigh numbers. The same qualitative trend in the evolution of the average Bejan number appear in the bench mark solution (figures 5-6). The obvious difference between the two solutions lies in the fact that the average Bejan number is close to one, according to our approach, while the same parameter varies significantly from 0.02 to 1 , according to the approach referenced in [1619], when the irreversibility distribution ratio value is set at $\varphi=10^{-4}$. This could be explained by the fact that irrespective of the Rayleigh number the irreversibility distribution ratio $\varphi$ is very small (see table 4) compared to that chosen for the benchmark test $\left(\varphi=10^{-4}\right)$. As a result, the total entropy generation due to fluid friction is negligible, which implies an average Bejan number close to one. This result confirms the commonly adopted hypothesis, which states that the viscous dissipation function is neglected in the energy transport equation. 


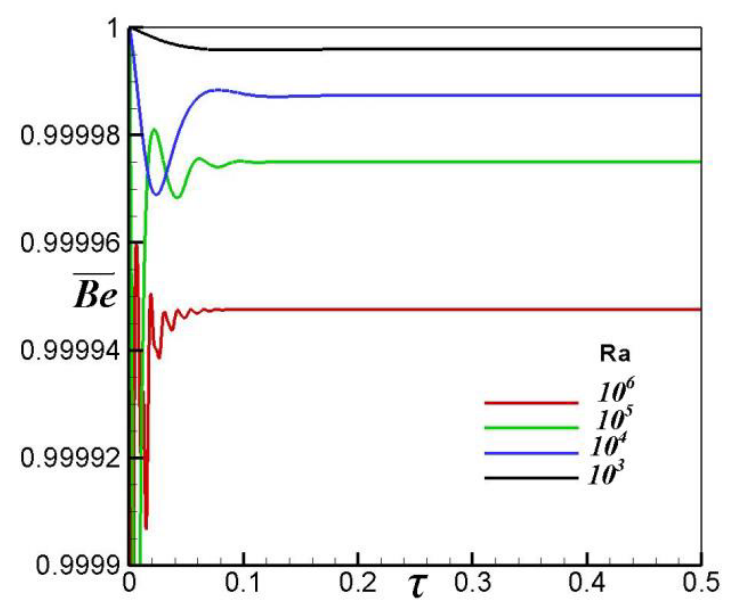

Fig. 5. Evolution of average Bejan number versus the Rayleigh numbers ( $\mathrm{Ra}$ ) according to our approach.

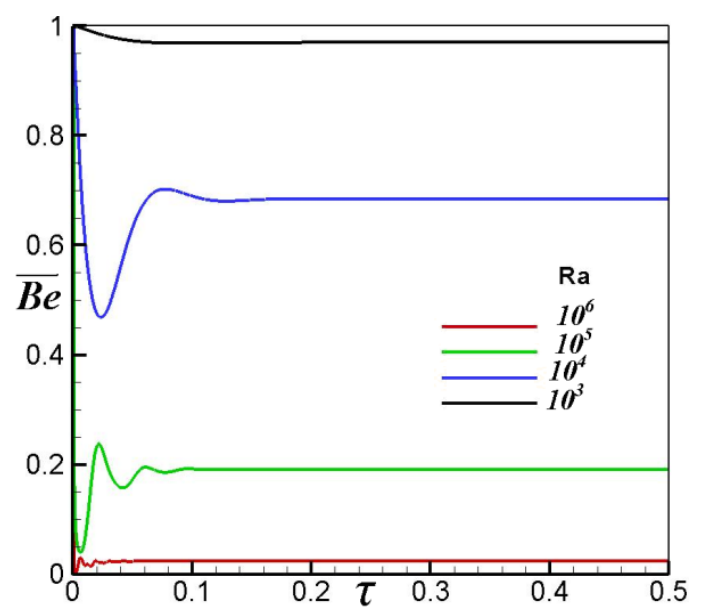

Fig. 6. Evolution of average Bejan number versus the Rayleigh numbers $(\mathrm{Ra})$ according to the approach referenced in [16-19], $(\varphi=10-4)$

\section{Conclusion}

In the present work, details are given of a new approach used to obtain an accurate determination of the entropy generation for unsteady natural convection in a square cavity with differentially heated sidewalls. The conclusions are summarized as follows:

1 From the mathematical point of view, it is clear that the characteristic length $\mathrm{L}$ is proportional to the Rayleigh number and then cannot be assumed as constant, especially if this number varies quite considerably.

2 The irreversibility distribution ratio number varies significantly according to the Rayleigh number and hence it can, in no case, be considered as a constant.
3 One and only one value of the irreversibility distribution ratio $\varphi$ corresponds to each fluid characterized by its Prandtl and Rayleigh numbers.

4 The irreversibility distribution ratio $\varphi$ is proportional to the inverse of the characteristic length L squared, and thus its value decreases when the characteristic length L (or Rayleigh number) increases.

5 The values of the irreversibility distribution ratio $\varphi$ proposed by different authors are being overestimated resulting often lead to an oversizing of the heat transfer processes equipment.

6 Though the present results are restricted to natural convection in confined spaces of limited Prandtl number and Rayleigh numbers, the procedure is general and can be equally applied to various problems including mixed convection, nanofluids flows, turbulent flows, and so on.

\section{ACKNOWLEDGMENTS}

The authors like to express their thankfulness to the Computational Fluid Dynamics Laboratory of the Mechanical Engineering Department, of Istanbul Medeniyet University, Turkey for having provided computer facilities during this work.

\section{NOMENCLATURE}

Be Bejan number

g Gravitational acceleration, $\mathrm{ms}^{-2}$

Gr Grashof number $\left(=\mathrm{g} \beta \Delta \mathrm{TL}^{3} / \mathrm{v}^{2}\right)$

$\mathrm{k}$ Thermal conductivity, $\mathrm{Wm}^{-1} \mathrm{~K}^{-1}$

L Length of the square cavity, $\mathrm{m}$

Pr Prandtl number, $(=v / \alpha)$

$\mathrm{Ra}$ Rayleigh number, $(=\mathrm{Gr}$ Pr)

$\mathrm{S}_{\text {gen }}$ Entropy generation, $\mathrm{Wm}^{-3} \mathrm{~K}^{-1}$

$\mathrm{t}$ Time, $\mathrm{s}$

$\mathrm{T}$ Absolute temperature, $\mathrm{K}$

T0 Bulk temperature $(\mathrm{Th}+\mathrm{Tc}) / 2), \mathrm{K}$

$\mathrm{u}, \mathrm{v}$ Velocity components in $\mathrm{x}, \mathrm{y}$ directions, $\mathrm{m} \mathrm{s}^{-1}$

$\mathrm{U}, \mathrm{V}$ Dimensionless velocities in $\mathrm{X}$ and $\mathrm{Y}$ direction

\section{$\vartheta \quad$ System volume}

$\mathrm{x}, \mathrm{y}$ Dimensional Cartesian coordinates, $\mathrm{m}$

X, Y Dimensionless Cartesian coordinates

\section{Greek symbols}

$\alpha \quad$ Thermal diffusivity, $\mathrm{m}^{2} \mathrm{~s}^{-1}$

$\beta \quad$ Thermal expansion coefficient, $\mathrm{K}^{-1}$

$\Delta \mathrm{T} \quad$ Temperature difference ((Th-Tc), $\mathrm{K}$

$\Theta \quad$ Dimensionless temperature

$v \quad$ Kinematic viscosity, $\mathrm{m}^{2} \mathrm{~s}^{-1}$

$\mu \quad$ Dynamic viscosity, $\mathrm{kg} \mathrm{m}^{-1} \mathrm{~s}^{-1}$

$\varphi \quad$ Irreversibility distribution ratio 
$\rho \quad$ Density, $\mathrm{kg} \mathrm{m}^{-3}$

$\tau \quad$ Dimensionless time

$\psi \quad$ Stream function, $\mathrm{m}^{2} \cdot \mathrm{s}^{-1}$

$\Psi \quad$ Dimensionless stream function

$\omega \quad$ Vorticity, $\mathrm{s}^{-1}$

$\Omega \quad$ Dimensionless vorticity

\section{Subscripts \\ ff Fluid friction \\ th Heat transfer}

\section{References}

1. Bejan, A., Entropy Generation through Heat and Fluid Flow, Wiley, New York (1982).

2. A. Bejan, Entropy generation minimization, The new thermodynamics of finite size devices and finite time processes, Journal of Applied Physics 79, 1191, (1996).

3. H.F. Oztop, K. Al-Salem, A review on entropy generation in natural and mixed convection heat transfer for energy systems, Renewable and Sustainable Energy Reviews, Vol 16(1) :pp911920, (2012).

4. A. Sciacovelli, V. Verda, E. Sciubba, Entropy generation analysis as a design tool-A review, Renew. Sust. Energ. Rev. 43, 1167-1181, (2015).

5. K. Kumar, R. Kumar, R. Singh Bharj, Entropy generation analysis due to heat transfer and nanofluid flow through microchannels: a review, Int. J. Exergy, Vol. 31(1) :pp49-86, (2020).

6. M. Cai, P. Cui, Y. Qin, D. Geng, Q. Wei, X. Wang, D. Yang, G. Zhang, Entropy Generation Methodology for Defect Analysis of Electronic and Mechanical Components-A Review, Entropy, Vol. 22(254) :pp1-19, (2020).

7. A. Hussein, K. Lioua, R. Chand, S. Sivasankaran, R. Nikbakhti, D. Li, B. Naceur and B. Habib, Three-dimensional unsteady natural convection and entropy generation in an inclined cubical trapezoidal cavity with an isothermal bottom wall, Alexandria Engineering Journal (55) :pp741-755, (2016).

8. H.F. Oztop, M. A. Almeshaal, L. Kolsi , M. M. Rashidi and M. E. Ali, Natural Convection and Irreversibility Evaluation in a Cubic Cavity with Partial Opening in Both Top and Bottom Sides, Entropy 21(116), (2019).

9. Shavik, S. M. Nasim Hassan, M. Monjur Morshed, A. K. M. Quamrul Islam, Natural convection and entropy generation in a square inclined cavity with differentially heated vertical walls. Procedia Engineering (90) :pp557-562, (2014).

10. O. Yejjer, L. Kolsi, Abdullah A.A.A. Al-Rashed, A. Aydi, M. N.Borjini1, H. Ben Aissia1, Numerical analysis of natural convection and entropy generation in a $3 \mathrm{D}$ partitioned cavity, International Journal of Heat and Technology 35 (4) :pp933-943, (2017).

11. H. M. Jassim, F. H. Ali, Q. R. Al-Amir, H. K. Hamzah, S. O. W. Khafaji, Entropy Generation Analysis of a Natural Convection inside a
Sinusoidal Enclosure with Different Shapes of Cylinders, Frontiers in Heat and Mass Transfer 12(22), (2019).

12. S. M. Seyyedi, A.S. Dogonchi, M. HashemiTilehnoee, M. Waqas, D. D. Ganji, Investigation of entropy generation in a square inclined cavity using control volume finite element method with aided quadratic Lagrange interpolation functions, International Communications in Heat and Mass Transfer (110) :pp104-398, (2020).

13. L. B. Erbay, Z. Altaç, B. Sülüş, An Analysis Of The Entropy Generation In A Square Enclosure, Entropy (5) :pp496-505, (2003).

14. L. B. Erbay, Z. Altaç, B. Sülüş, Entropy generation in a square enclosure with partial heating from a vertical lateral wall, Heat and Mass Transfer (40) :pp909-918, (2004).

15. V. Mani Rathnam, M. Roy \& T. Basak, Analysis of entropy generation during natural convection in tilted triangular enclosures with various base angles, Numerical Heat Transfer, Part A: Applications 69(12) :pp1332-1354, (2016).

16. M. Magherbi, H. Abbassi, and A. B. Brahim, Entropy Generation at the Onset of Natural Convection, Int. J. Heat Mass Transfer (46) :pp3441-3450, (2003).

17. G.M. Ilis, M. Mobedi, B. Sunden, Effect of aspect ratio on entropy generation in a rectangular cavity with differentially heated vertical walls. International Communication in Heat Mass Transfer 35(6) :pp96-703, (2008).

18. Rejane De C. Oliveski, Mario H. Macagnan, Jacqueline B. Copetti, Entropy generation and natural convection in rectangular cavities, Applied Thermal Engineering Vol (29):pp1417-1425, (2009).

19. M. Bouabid, M. Magherbi, N. Hidouri, A. Ben Brahim, Entropy Generation at Natural Convection in an Inclined Rectangular Cavity, Entropy (13) : pp1020-1033, (2011).

20. P. Karki, D. A. Perumal, A.K. Yadav, Comparative studies on air, water and nanofluids based Rayleigh-Benard natural convection using lattice Boltzmann method:CFD and exergy analysis, J Therm Anal Calorim, (2021).

21. H. Khorasanizadeh, J. Amani, M. Nikfar, Numerical investigation of $\mathrm{Cu}$-water Nano fluid natural convection and entropy generation within a cavity with an embedded conductive baffle, Scientia Iranica Transactions F, Nanotechnology 19(6) :pp1996-2003, (2012).

22. Bejan, A., Convection Heat Transfer, 4th Edition, John Wiley \& Sons, Chap (1) :p17, Inc. Hoboken, New Jersey (2013).

23. T. Kawamura, H.Takami, K. Kuwahara, New higher order upwind scheme for incompressible Navier-Stokes equations. Ninth International Conference on Numerical Methods in Fluid Dynamics. Lecture Notes in Physics, Vol (218) : pp291-295, (1985).

24. G. de Vahl Davis, Natural Convection of Air in a Square Cavity: A Benchmark Numerical Solution, 
Int. J. Numer. Meth. Fluids, vol(3) :pp249-264, (1983).

25. P. Nithiarasu, K. N. Seetharamu, T. Sundararajan, Natural Convective Heat Transfer in a Fluid Saturated Variable Porosity Medium, Int. J. Heat Mass Transfer, vol(40) :pp3955-3967, (1997).

26. D. Santhosh Kumar, A. K. Dass, A. Dewan, Analysis of Non-Darcy Models for Mixed Convection in a Porous Cavity Using a Multigrid Approach, Numerical Heat Transfer, Part (A), Applications 56(8) :pp685-708, (2009).

27. Incropera, F. P., Dewitt, D. P., Bergman, T. L., and Lavine, A. S., Fundamentals of heat and mass transfer, 6th edition, John Wiley \& Sons, (2007). 